\title{
VIVÊNCIA DE ACADÊMICOS DE ENFERMAGEM NA OPERACIONALIZAÇÃO DE ENSAIO CLÍNICO RANDOMIZADO: RELATO DE EXPERIÊNCIA
}

EXPERIENCE OF NURSING ACADEMICS IN THE RANDOMIZED CLINICAL TEST OPERATION: CASE STUDIES

\section{LA EXPERIENCIA DE ACADÉMICOS DE ENFERMERÍA EN EL FUNCIONAMIENTO DE ENSAYO CLÍNICO RANDOMIZADO: ESTUDIOS DE CASO}

Daniel Vinicius Alves Silva ${ }^{1}$, Carolina Amaral Oliveira ${ }^{1}$, Patrícia Oliveira Silva ${ }^{1}$, Tânia Couto Machado Chianca ${ }^{2}$, Diego Dias de Araújo ${ }^{3}$.

\section{RESUMO}

Objetivo: Relatar a experiência e atividades de acadêmicos de enfermagem na operacionalização de um ensaio clínico randomizado. Métodos: Trata-se de um estudo descritivo na modalidade de relato de experiência, no qual relatou-se a experiência e atividades de acadêmicos de enfermagem na operacionalização de um ensaio clínico randomizado conduzido em pacientes criticamente enfermos, internados na unidade de terapia intensiva de um hospital filantrópico do norte de Minas Gerais, Brasil. Resultados: As funções dos acadêmicos foram realizar a alocação das intervenções que os pacientes recebiam, mediante uma lista randomizada e aleatorizada; coletar dados sob a supervisão de um dos pesquisadores e digitar os dados em programa estatístico. 0 método empregado é considerado ideal, pois possibilita que os acadêmicos e profissionais de enfermagem reflitam sobre a prática de enfermagem científica e não intuitiva, ao se testar e validar cientificamente intervenções que são implementadas no cotidiano da enfermagem, muitas vezes de maneira empírica. Conclusão: A experiência na operacionalização desse tipo de estudo, por acadêmicos de enfermagem, contribui para formação profissional, ao proporcionar o desenvolvimento do pensamento crítico, com vista à resolução de problemas presentes na prática clínica de enfermagem.

Descritores: Ensaio clínico controlado; Enfermagem; Pesquisa em enfermagem clínica; Aprendizagem baseada em problemas.

\begin{abstract}
Objective: To report the experience and activities of nursing students in the operationalization of a randomized clinical test. Methods: This is a case study, descriptive, in which the experience and activities of nursing students in the operationalization of a randomized clinical test conducted in critically ill patients hospitalized in the intensive care unit of a philanthropic hospital in the north of Minas Gerais, Brazil. Results: The functions of the academics were to perform the allocation of the interventions that the patients received through a randomized and aleatory list; it collected data under the supervision of one of the researchers and enter the data into a statistical program. The method applied is ideal, since it allows nursing academics and professionals to reflect on a scientific and non-intuitive nursing practice, by scientifically testing and validating interventions implemented in the daily routine of nursing, often empirically. Conclusion: The experience in the operationalization of this type of study by nursing undergraduates contributes to professional training by providing the development of critical thinking, in order to solve problems present in clinical nursing practice.
\end{abstract}

Descriptors: Controlled clinical test; Nursing; Clinical nursing research; Problem-based learning.

\section{RESUMEN}

Objetivo: Presentar la experiencia y las actividades de los estudiantes de enfermería en la operación de una prueba clínica aleatorizada. Métodos: Se trata de un estudio de caso, descriptivo, en el cual la experiencia y las actividades de los estudiantes en la operacionalización de un teste clínico randomizado realizado en pacientes críticamente enfermos ingresados en la unidad de cuidados intensivos de un hospital filantrópico en el norte de Minas Gerais, Brasil. Resultados: Las funciones de los académicos fueron realizar la asignación de las intervenciones que los pacientes recibían mediante una lista aleatorizada; recoger datos bajo la supervisión de uno de los investigadores e introducir los datos en el programa estadístico. El método empleado es considerado ideal, ya que posibilita a los académicos y profesionales de enfermería reflexionar acerca de una práctica de enfermería científica y no intuitiva, al probar y validar científicamente las intervenciones que se implementan en el cotidiano de la enfermería, muchas veces de forma empírica. Conclusión: La experiencia en la operacionalización de este tipo de estudio por académicos de enfermería contribuye a la formación profesional al proporcionar el desarrollo del pensamiento crítico, con miras a la resolución de problemas presentes en la práctica clínica de enfermería.

Descriptores: Prueba clínica controlada; Enfermería; Investigación en enfermería clínica; Aprendizaje basada en problemas.

${ }^{1}$ Graduado em Enfermagem pela Universidade Estadual de Montes Claros. ${ }^{2}$ Graduada em Enfermagem. Pós-Doutora pelo Center for Nursing Classification do College of Nursing -The University of lowa. Docente na Universidade Federal de Minas Gerais. ${ }^{3}$ Graduado em Enfermagem. Doutor em Enfermagem pela Universidade Federal de Minas Gerais. Docente na Unimontes.

\section{Como citar este artigo:}

Silva DVA, Oliveira CA, Silva PO, et al. Estratégia para Intensificar a Prática Baseada em Evidência na Formação de Acadêmicos de Enfermagem: Relato de Experiência. Revista de Enfermagem do Centro-Oeste Mineiro. 2018;8:e1784. [Access Available in: DOI: http://dx.doi.org/10.19175/recom.v9i0.1784 


\section{INTRODUÇÃO}

A construção do conhecimento deve ser baseada em um diálogo permanente entre todos os participantes do processo de ensino e aprendizagem, estando dentro ou fora do espaço físico acadêmico, proporcionando, assim, uma relação entre o teórico e o prático ${ }^{(1)}$.

A pesquisa em saúde é fonte produtora dessa construção, uma atividade que busca novas descobertas científicas, contribuindo para a qualidade de novos estudos, além de sua execução tender a resultar na incorporação de novos conhecimentos no cuidado em saúde. Nesse sentido, as intervenções a serem implementadas na prática clínica devem ser baseadas em pesquisas, em evidências científicas $^{(2-4)}$.

Destaca-se no desenvolvimento de fortes evidências científicas, a pesquisa clínica. Ela é recente, caracterizada pelos avanços de boas práticas clínicas, consolidadas nos Estados Unidos em 1988, pelo Food and Drug Administration (FDA), o qual dispõe normas, orientações éticas e científicas para o progresso do estudo clínico ${ }^{(3,5)}$. No Brasil, existe após ser aprovada a Resolução do Conselho Nacional de Saúde (CNS) 196/96, revisada em 2012, com a Resolução CNS 466/12, em que regula as pesquisas com seres humanos em todo território nacional.

Estudo clínico, pesquisa clínica ou ensaio clínico são sinônimos e investigações com seres humanos sadios ou doentes estão envolvidas ${ }^{(3)}$. Trata-se de um estudo sistemático que segue métodos nos quais os resultados podem gerar novos conhecimentos sobre procedimentos, medicamentos ou intervenções que melhorem a saúde das pessoas ${ }^{(3,6-8)}$.

Nos estudos clínicos, um grupo de interesse que está utilizando uma nova terapia (grupo intervenção) é acompanhado e comparado com outro que faz uso de uma terapia já padronizada na prática clínica ou um placebo (grupo controle). O estudo clínico randomizado (ECR) é considerado o método mais confiável para analisar o efeito de um tratamento ou intervenção ${ }^{(7)}$.

A alocação dos sujeitos de pesquisa nos grupos do ECR pode ser realizada de forma aleatória ou não aleatória ${ }^{(6)}$. A aleatorização possibilita que as comparações dos tratamentos não sejam afetadas pela seleção, consciente ou não, dos participantes com uma característica específica para um tratamento particular. Assim, tal estratégia reduz a chance de parcialidades ou tendências e, consequentemente, de fontes de viés de seleção ${ }^{(9)}$.

Assim como em diversas áreas do conhecimento, na enfermagem, observa-se uma carência desse tipo de estudo, principalmente dado o campo de atuação recente, o que tem dificultado a tomada de decisão na prática clínica ${ }^{(7)}$. A ausência de provas científicas suficientemente fortes pode influenciar em um julgamento clínico restrito a respeito de uma determinada situação, como na eficácia de certo medicamento ou qual a melhor intervenção a ser aplicada. Diante disso, é fundamental o desenvolvimento de pesquisas clínicas randomizadas que possibilitem avaliar uma intervenção em relação a sua efetividade, segurança e custo-benefício ${ }^{(2,4)}$.

Destaca-se que o estudo clínico ainda é pouco explorado e abordado na graduação em enfermagem. No Brasil, os primeiros estudos iniciaram na década de 80 , envolvendo a definição de um determinado problema, a busca e avaliação crítica das evidências disponíveis, implementação das evidências na prática e avaliação dos resultados obtidos, sendo que a maioria dos estudos disponíveis são em literatura internacional ${ }^{(4,7)}$.

No campo da enfermagem, tem sido desenvolvido um ECR, cujo objetivo é avaliar o efeito de intervenções de enfermagem (lágrima artificial em gel e lágrima artificial líquida) na prevenção de olho seco, em pacientes internados em Unidade de Terapia Intensiva (UTI) geral de adultos de um hospital filantrópico do norte de Minas Gerais, Brasil.

O olho seco é uma síndrome que está relacionada à deficiência na produção da lágrima ou o aumento em sua evaporação, influenciando assim, a manutenção da superfície córneoconjuntiva $^{(10)}$. De acordo com a NANDA International, Inc. (NANDA-I) ${ }^{(11)}$, o conceito do diagnóstico de enfermagem de risco de olho seco é "risco de desconforto ocular e danos à córnea e conjuntiva devido à quantidade reduzida ou qualidade de lágrimas para umedecer o olho".

Pacientes internados em UTI podem apresentar condições de riscos elevadas para o desenvolvimento de olho seco, uma vez que, na maioria das vezes, estes estão sedados, em coma, com ventilação mecânica (VM), em uso de diversos medicamentos e mecanismos de proteção ocular comprometidos ${ }^{(12-13)}$. Enfatiza-se que, devido à priorização da assistência a sistemas fisiológicos considerados vitais como o 
cardiovascular, respiratório e neurológico, os cuidados oculares normalmente são tratados secundariamente ${ }^{(12)}$.

Estudo recente ${ }^{(12)}$ apontou que o olho seco é um problema comum em pacientes internados em UTIs de adultos, apresentando uma incidência de $53 \%$. Desta maneira, os cuidados específicos para a prevenção do olho seco são de suma importância pois, podem reduzir a probabilidade de desconfortos e alterações oculares em pacientes criticamente enfermos, tanto durante a internação, quanto após a alta da UTI.

Justifica-se este relato de experiência, uma vez que, ainda que a pesquisa clínica, no geral, não seja muito conhecida e compreendida pelos profissionais de enfermagem, entende-se ser fundamental que discussões sobre a temática, desde a graduação, sejam incitadas. Além disso, pretende-se contribuir para estimular a compreensão a respeito do delineamento ECR e sua importância para crescimento e fortalecimento da prática de enfermagem baseada em fortes evidências científicas.

Diante de nossa experiência em ECR e, por ser este tipo de estudo relevante a ser incrementado na enfermagem, objetiva-se, no presente estudo, relatar a experiência e atividades dos acadêmicos de enfermagem na operacionalização de um ECR, destacando a importância deste tipo de delineamento.

\section{MÉTODOS}

Trata-se de um estudo descritivo na modalidade de relato de experiência. Esse tipo de estudo possibilita conhecer profundamente a experiência descrita, mais do que ser uma narração precisa acerca de uma determinada atividade. Além disso, proporciona a reflexão a respeito do conteúdo abordado ${ }^{(14)}$.

A experiência a ser relatada está relacionada a um de ECR, desenvolvido por uma equipe de pesquisa com financiamento do Conselho Nacional de Desenvolvimento Científico e Tecnológico (CNPq). Fizeram parte da equipe, um aluno de doutorado, uma pesquisadora e três acadêmicos de iniciação científica de um curso de graduação em Enfermagem do norte de Minas Gerais, Brasil.

A coleta de dados do estudo foi realizada no período entre 14 de janeiro de 2016 e 14 de março de 2017, em um hospital filantrópico de cuidados terciários. Com 275 leitos, este é classificado como de grande porte. O hospital possui participação ativa no sistema de urgência do norte de Minas Gerais, sendo referência para urgências clínicas, traumatológicas e cirúrgicas, bem como para a realização de cirurgias eletivas. Atualmente, são disponibilizados à comunidade, 10 leitos de tratamento intensivo destinados a pessoas adultas.

A seleção dos acadêmicos para a participação no estudo ocorreu antes de iniciar a pesquisa de campo. Para tal, seguiram-se alguns critérios como: obter bom desempenho acadêmico, disciplina, comprometimento, manifestação do desejo e disponibilidade para participar do projeto. Os mesmos encontravamse matriculados no quarto período do curso de graduação em Enfermagem e dedicaram ao estudo uma carga horária semanal de 20 horas.

As funções desempenhadas pelos acadêmicos englobavam o controle e alocação das intervenções que cada paciente recebia, através de uma lista randomizada e aleatorizada; coleta de dados sob a supervisão de um dos pesquisadores e digitação dos dados em programa estatístico.

Destaca-se que, na coleta de dados, além das variáveis sociodemográficas e clínicas, para avaliação do volume lacrimal, foi utilizado o teste de Schirmer I, que consiste na instalação de uma tira de Whatman número 41 ou 50 , com cinco milímetros $(\mathrm{mm})$ de largura e $35 \mathrm{~mm}$ de comprimento, com a ponta dobrada (cerca de cinco $\mathrm{mm}$ ), encaixada no fundo do saco palpebral inferior na porção temporal (canto externo da pálpebra inferior). Após cinco minutos, a fita era retirada, medida e anotada a extensão da parte umedecida. Para avalição corneana, foi instalada uma gota de fluoresceína em cada olho do paciente e, após um a dois minutos, sob condições de baixa luminosidade, a córnea era examinada com auxílio de um oftalmoscópio com filtro de luz azul-cobalto e lupa, para melhor visualização de possíveis alterações corneanas ${ }^{(10)}$.

O estudo que fez parte do relato de experiência está em conformidade com a Resolução 466/12, que dispõe sobre a pesquisa com seres humanos. O projeto foi encaminhado ao Comitê de Ética e Pesquisa da Universidade Federal de Minas Gerais e obteve parecer favorável sob o protocolo CAAE 50711915.8.0000.5149.

\section{RESULTADOS E DISCUSSÃO}

Após a seleção dos acadêmicos, foram estabelecidas algumas etapas de capacitação. No primeiro momento, a capacitação consistiu em 
leitura prévia de artigos e textos sobre as temáticas, explanação teórica sobre olho seco, avaliação ocular e sobre o delineamento de pesquisa ensaio clínico randomizado. A capacitação dos acadêmicos pelo professor pesquisador foi fundamental, pois possibilitou a aproximação dos integrantes com a temática a ser abordada, uma vez que muitos a desconhecia, além do aprofundamento do próprio delineamento do estudo, já que este é explorado e abordado de maneira superficial na graduação em enfermagem.

$\mathrm{Na}$ segunda etapa, houve discussões em relação aos instrumentos de coleta de dados, ocasião em que foram trabalhadas as variáveis que os compunham, os testes clínicos (teste de fluoresceína e teste de Schirmer I) que eram realizados durante a avaliação ocular, além de capacitação para manuseio do programa estatístico no qual os dados eram digitados. Para este estudo, escolheu-se trabalhar com o programa Epi Info, versão 3.5.1, devido ser de fácil manejo e por possibilitar, após a dupla digitação, a verificação da consistência das informações. Evidencia-se que é necessário que a geração de qualquer dado, em uma pesquisa, seja fidedigna, para que esse possa auxiliar na tomada da decisão correta por parte de outros profissionais $^{(8,15)}$.

Esta etapa oportunizou a padronização das informações entre a equipe, além da aproximação, entendimento e familiarização com as ferramentas de trabalho que foram utilizadas ao longo do estudo.

Concluída a capacitação dos acadêmicos, iniciaram-se os treinamentos com as equipes de profissionais do hospital onde o estudo foi conduzido. Foram treinadas quatro equipes, sendo que, cada uma delas era composta por um enfermeiro e cinco técnicos de enfermagem, que trabalham em escala, na modalidade 12 horas de plantão e 36 horas de folga, totalizando quatro enfermeiros e vinte técnicos de enfermagem. Os treinamentos ocorreram em dois dias consecutivos, durante as trocas de plantão. Esses foram ministrados pelo professor pesquisador, com o auxílio dos acadêmicos. O treinamento consistiu na explanação do problema de estudo; critérios de inclusão para participação dos pacientes na pesquisa; termo de consentimento, uma vez que os enfermeiros colaboravam, abordando os familiares para obtenção do consentimento; e a maneira de aplicação de cada uma das intervenções pelos técnicos de enfermagem.

A etapa de capacitação da equipe foi essencial para a formação do vínculo entre acadêmicos e o professor pesquisador, e os profissionais que atuam na prática de terapia intensiva do local de estudo, possibilitando garantir a credibilidade do estudo, adesão e participação (uma vez que esta era voluntária) em cada uma das funções atribuídas.

Iniciado o estudo propriamente dito, após a captação do termo de consentimento pelos enfermeiros, os mesmos entravam em contato, através de uma central de informações, que foi criada, pelos acadêmicos, com o auxílio de um aplicativo telefônico, e que não contava com a participação e presença do professor pesquisador devido ao mascaramento do estudo. Através da central, os enfermeiros eram informados, pelos acadêmicos, a respeito de qual intervenção seria implementada pelos técnicos de enfermagem.

Por se tratar de um ECR duplo-cego, no qual $o$ pesquisador e $\mathrm{o}$ paciente não têm conhecimento da intervenção que está sendo implementada ${ }^{(16)}$, neste estudo, a lista randomizada e aleatorizada com as intervenções foi enviada diretamente, por um estatístico de outro estado, aos acadêmicos e estes foram responsáveis por alocar cada paciente conforme a intervenção sorteada. Essa estratégia é essencial para se evitar possíveis vieses de aferição, uma vez que o conhecimento da intervenção que está sendo implementada pode tendenciar o resultado encontrado durante a avaliação em relação à presença ou ausência do desfecho; ou viés de seleção, por se alocar o participante em alguma das intervenções devido ao seu perfil clínico, levando em consideração a hipótese de que aquela intervenção pode ser a mais eficaz para solucionar o problema.

A colaboração na etapa supracitada possibilitou que os acadêmicos desenvolvessem a percepção da necessidade de planejamento e organização relacionados ao rigor metodológico do tipo de estudo. Além disso, em consonância com as Diretrizes Curriculares Nacionais para o curso de graduação em Enfermagem, permitiu-se o aperfeiçoamento intelectual e a capacitação na busca da autonomia profissional.

Após os enfermeiros receberem a notificação, através da central de informações, de qual intervenção seria implementada, a mesma era, então, separada pelos mesmos e acondicionada em um envelope pardo, com o 
intuito também de manter o mascaramento da intervenção.

A função dos técnicos de enfermagem era administrar a intervenção, conforme a randomização, em dois momentos do dia: às 08:00 horas da manhã e às 20:00 horas da noite, durante cinco dias consecutivos. A certificação da realização do procedimento pelos técnicos era realizada através de um instrumento de coleta de dados, no qual os mesmos deviam checar se foi ou não implementado o cuidado nos horários supracitados e se ocorreu alguma inconformidade, o que levaria à perda e saída do paciente do estudo.

Observou-se que, apesar do cansaço físico e muitas vezes psicológico, devido às diversas demandas e situações as quais os profissionais que trabalham em UTI estão expostos, a participação e o envolvimento no estudo foi uma oportunidade de se capacitarem e atuarem em um tipo de pesquisa ainda desconhecida para muitos e de grande relevância para a enfermagem. Tais se sentiam valorizados, pois sabiam da importância de seu papel, tanto para condução do estudo, quanto na implementação dos cuidados a serem prestados. Além disso, foi notório o reconhecimento de outras categorias profissionais em relação ao estudo que estava sendo desenvolvido.

Diariamente, durante os cinco dias consecutivos, o professor pesquisador, com o auxílio de um dos acadêmicos, compareciam ao hospital para realização da coleta de dados e avaliação ocular. Para avaliação do volume lacrimal, foi utilizado o teste de Schirmer I e, para identificação da presença ou ausência de lesão de córnea, o teste de fluoresceína. Após a coleta, os dados eram duplamente digitados no programa Epi Info, versão 3.5.1.

A participação dos acadêmicos na etapa de coleta de dados proporcionou vivência clínica e um maior conhecimento da área hospitalar, principalmente sobre o perfil de pacientes em terapia intensiva. Pode-se notar que esta é uma área que exige dos profissionais, responsabilidade, agilidade, destreza, rápido raciocínio clínico e capacidade de tomar decisões imediatas, uma vez que, nas UTIs, presta-se assistência a pacientes acometidos por insuficiências orgânicas graves ou sob risco de desenvolvê-las, podendo, estes, mudarem rapidamente o perfil de estabilidade clínica.

Ensinar conceitos e ideias pela apropriação ou a apreensão deste, por parte dos discentes, demanda a criação e o exercício de uma séria disciplina. Assim, o ser docente deve ser capaz de perceber, entender, analisar e acompanhar as mudanças que ocorrem no decorrer do processo ensino aprendizagem ${ }^{(17-18)}$. A partir da prática cognoscente e da pesquisa científica, os discentes se formam sujeitos cada vez mais críticos e obtém conhecimento especifico e estruturado sobre um assunto preciso ${ }^{(15,18)}$.

A experiência aponta para a relevância da realização deste tipo de estudo por enfermeiros e participação de acadêmicos de enfermagem devido à influência desta experiência extracurricular no aperfeiçoamento da formação acadêmica e profissional. O método empregado é considerado ideal, tendo em vista que possibilita que os profissionais e acadêmicos de enfermagem reflitam sobre uma prática de enfermagem científica e não intuitiva, ao se testar e validar cientificamente intervenções que são implementadas no cotidiano da enfermagem, muitas vezes de forma empírica.

\section{CONCLUSÃO}

A experiência vivenciada, durante a graduação em enfermagem, em pesquisa clínica, foi de suma importância. Os momentos de discussões acerca do tema e a participação na execução das diversas etapas do estudo proporcionaram aos acadêmicos que adquirissem novos conhecimentos, os quais, provavelmente, não seriam contemplados com profundidade durante a formação acadêmica. Tal, tende a impactar em uma futura prática profissional de maior qualidade, ao possibilitar aos mesmos uma base para desenvolver pesquisas científicas com delineamento robusto, colaborando para o crescimento e fortalecimento da enfermagem enquanto ciência.

Destaca-se a oportunidade de participar de um estudo em que o cuidado prático é testado e validado cientificamente com consequentes resultados positivos na assistência de enfermagem, ao se implementar, na prática clínica, cuidados baseados em forte evidência científica.

Nota-se que, apesar de o delineamento ser complexo e de difícil manejo, o profissional enfermeiro é apto para planejar e realizar um estudo desse porte, e que a inserção dos acadêmicos, nesse tipo de pesquisa, é importante, pois impacta no desenvolvimento de um profissional ainda mais crítico e capaz de 
buscar soluções para os diversos problemas presentes na prática cotidiana da enfermagem.

Por fim, esses novos saberes são indispensáveis diante dos avanços tecnológicos atuais, que exigem constante capacitação e aprimoramento técnico-científico, a fim de que os profissionais desenvolvam competências e sejam capazes de transportar o conhecimento científico teórico para a prática do cuidado.

\section{REFERENCES}

1 - Freitas DA, Santos EMS, Lima LVS, Miranda LN, Vasconcelos EL, Nagliate PC. Saberes docentes sobre processo ensino-aprendizagem e sua importância para a formação profissional em saúde. Interface 2016 abr/jun;20(57):437-48. DOI: 10.1590/1807-57622014.1177

2 - Ministério da Saúde. Política Nacional de Ciência, Tecnologia e Inovação em Saúde. Brasília: Ministério da Saúde; 2008.

3 - Pedrolo E. Pesquisa clínica em enfermagem: contribuições para inovação tecnológica. Rev Min Enferm. 2012 jul/set;16(3):445-53. DOI: 141527622012000300017

4 - Vasques $\mathrm{Cl}$. A importância da pesquisa clínica para o avanço da enfermagem. $R$ Enferm Cent $O$ Min. 2016 jan/mar; 6(1):1-2. DOI: 10.19175/recom.v0i0.1389

5 - Danski MTR, Oliveira GLR, Pedrolo E, Lind J, Johann DA. Importância da prática baseada em evidências nos processos de trabalho do enfermeiro. Cienc Cuid Saude 2017 abr/jun;16(2):1-6.

DOI: $10.4025 /$ cienccuidsaude.v16i2.36304

6 - Pérez-Campos MA, Sánchez-García I, Pancorbo-Hidalgo PL. Knowledge, attitude and use of evidence-based practice among nurses active on the internet. Invest Educ Enferm. 2014 32(3):451-60. DOI: 10.1590/S012053072014000300010.

7 - Nedel WL, Silveira F da. Different research designs and their characteristics in intensive care. Rev Bras Ter Intensiva 2016 July/Sept;28(3):25660.DOI: $10.5935 / 0103-507 X .20160050$

8 - Mancuso ACB, Camey SA, Nunes LN, Hirakata VN, Guimarães LSP. Os principais delineamentos na Epidemiologia Ensaios Clínicos (Parte I). Rev HCPA. 2013;33(3/4):286-94. URI: http:// hdl.handle.net/10183/158318

9 - American Academy of Ophtalmology (AAO). Dry eye syndrome - Limited revision. San Francisco: Am Acad of Opht; 2011.

10 - Herdman TH, Kamitsuru S. NANDA International nursing diagnoses: Definitions \& classification, 2018-2020. New York, NY: Theime; 2017.

11 - Araújo DD, Almeida NG, Silva PMA, Ribeiro NS, Werli-Alvarenga A, Chianca TCM. Prediction of risk and incidence of dry eye in critical patients. Rev Latino-Am Enfermagem 2016 maio;24:1-8. DOI: 10.1590/1518-8345.0897.2689

12 - Güler EK, Eser I, Egrilmez S. Effectiveness of polyethylene covers versus carbomerdrops (Viscotears) to prevent dry eye syndrome in the criticallyill. J. Clin Nurs. 2011 July;20(13/14)191622.DOI: 10.1111/j.1365-2702.2010.03559.x

13 - Fonseca GGP, Parcianello MK. O enfermeiro na comissão de controle de infecção hospitalar na perspectiva ecossistêmica: relato de experiência. R Enferm Cent O Min. 2014 maio/ago;4(2):121421. DOI: $10.19175 /$ recom.v0i0.441

14 - González-Chordá VM, Maciá-Soler ML. Evaluation of the quality of the teaching-learning process in undergraduate courses in Nursing. Rev Latino-Am Enfermagem 2015 jul/ago;23(4):700-7. DOI: $10.1590 / 0104-1169.0393 .2606$

15 - Andrade CT, Magedanz AM, Escobosa DM, Tomaz WM, Santinho CS, Lopes TO, Lombardo V. The importance of a database in the management of healthcare services. Einstein 2012 July/Sept;10(3):360-5. DOI: $10.1590 /$ S167945082012000300018

16 - Dainesi SMG, Goldbaum M. Pesquisa clínica como estratégia de desenvolvimento em saúde. Rev Assoc Med Bras. 2012 jan/fev;58(1):2-6. DOI: 10.1590/S0104-42302012000100002

17 - Freitas MAO, Cunha ICKO, Batista SHSS, Rossit RAS. Teaching in health: Perceptions of graduates of a Nursing specialization course. Interface 2016 abr/jun;20(57):427-3. DOI: $10.1590 / 1807-$ 57622015.0391

18 - Araújo AML, Morais HCC, Vasconcelos HCA, Rabelo JC, Santos RXL, Holanda RL. Scientific research in nursing graduation and its importance in vocational training. Rev Enferm UFPE 2015;9(9):9180-7. DOI: 10.5205/reuol.787468950-4-SM.0909201504

Nota: Agência de fomento Conselho Nacional de Desenvolvimento Científico e Tecnológico (CNPq), processo 441971/2014-8.

Recebido em: 02/02/2017

Aprovado em: 17/05/2018

Endereço de correspondência:

Diego Dias de Araújo

Universidade Estadual de Montes Claros - Departamento de Enfermagem - Campus Universitário Prof. Darcy Ribeiro Avenida Dr. Ruy Braga, S/N, Vila Mauriceia, Prédio 6 (CCBS) 
CEP: 39401-089 - Montes Claros/MG - Brasil

E-mail: diego.dias1508@gmail.com 